\title{
Benchmarking of surgical complications in gynaecological oncology: prospective multicentre study
}

Matthew Burnell ${ }^{1}$, Rema lyer ${ }^{1}$, Aleksandra Gentry-Maharaj ${ }^{\star 1}$, Andy Nordin ${ }^{2}$, Robert Liston$^{1}$, Ranjit Manchanda ${ }^{1,12}$, Nagindra Das ${ }^{3}$, Rob Gornall ${ }^{4}$, Alice Beardmore-Gray ${ }^{1}$, Kathryn Hillaby ${ }^{4}$, Simon Leeson ${ }^{5}$, Anders Linder ${ }^{6}$, Alberto Lopes ${ }^{3}$, David Meechan, Tim Mould ${ }^{8}$, James Nevin ${ }^{9}$, Adeola Olaitan ${ }^{8}$, Barnaby Rufford ${ }^{6}$, Smruta Shanbhag ${ }^{10}$, Alexandra Thackeray ${ }^{7}$, Nick Wood ${ }^{11}$, Karina Reynolds ${ }^{12}$, Andy Ryan ${ }^{1}$, Usha Menon ${ }^{1}$ *joint

Affiliation

${ }^{1}$ Gynaecological Cancer Research Centre, Dept of Women's Cancer, Institute for Women's Health, University College London, Maple House, 149 Tottenham Court Road, London W1T 7DN

${ }^{2}$ East Kent Gynaecological Oncology Centre, Queen Elizabeth the Queen Mother Hospital, St Peter's Road, Margate, Kent CT9 4AN

${ }^{3}$ Dept of Gynaecological Cancer, Royal Cornwall Hospitals NHS Trust, Truro, Cornwall TR1 3LJ

${ }^{4}$ Dept of Gynaecological Oncology, Cheltenham General Hospital, Sandford Road, Cheltenham, Gloucestershire, GL53 7AN

${ }^{5}$ Dept of Obstetrics and Gynaecology, BetsiCadwaladr University Health Board, Penrhosgarnedd, Bangor, Gwynedd, North Wales, LL57 2PW

${ }^{6}$ Dept of Gynaecological Oncology, The Ipswich Hospital NHS Trust, Heath Road, Ipswich, Suffolk, IP4 5PD

${ }^{7}$ Public Health England, 5 Old Fulwood Road, Sheffield S10 3TG

${ }^{8}$ Dept of Gynaecological Oncology, University College London Hospital NHS Foundation Trust, 2nd Floor North, 250 Euston Road, London NW1 2PG

${ }^{9}$ Pan Birmingham Gynaecological Cancer Centre, Birmingham City Hospital, Dudley Road, Birmingham, West Midlands, B18 7QH

${ }^{10}$ Dept of Gynaecological Oncology, Glasgow Royal Infirmary, 16 Alexandra Parade, Glasgow G31 2ER 
${ }^{11}$ Dept of Gynaecological Oncology, Lancashire Teaching Hospitals NHS Foundation trust, Royal Preston Hospital, Sharoe Green Lane, North Fulwood, Preston Lancashire PR2 9HT

${ }^{12}$ Dept of Gynaecological Cancer, Barts Cancer Centre, Barts and the London NHS Trust, West Smithfield, London EC1A 7BE

*Corresponding author: Usha Menon, Head Gynaecological Cancer Research Centre, Department of Women's Cancer, $1^{\text {st }}$ Floor Maple House, 149 Tottenham Court Road, University College London, London W1T 7DN, United Kingdom

Email address: u.menon@ucl.ac.uk

Telephone: +44 203447 2108/2112

Fax: +442034472129

Benchmarking in gynaecological oncology 


\section{Abstract}

Objective: To explore the impact of risk-adjustment on surgical complication rates (CRs) for benchmarking gynaecological-oncology centres.

Design: Prospective cohort study

Setting: Ten UK accredited gynaecological-oncology centres

Population: Women undergoing major surgery on a gynaecological-oncology operating list

Methods: Patient co-morbidity, surgical procedures and intraoperative (IntraOp) complications were recorded contemporaneously by surgeons for 2948 major surgical procedures. Postoperative (PostOp) complications were collected from hospitals and patients. Risk-prediction models for IntraOp and PostOp complications were created using penalised (lasso) logistic regression using over 30 potential patient/surgical riskfactors.

Main outcome measures: Observed and risk-adjusted IntraOp and PostOp CRs for individual hospitals were calculated. Benchmarking using colour-coded funnel plots and observed-to-expected ratios was undertaken.

Results: Overall, IntraOp CR was $4.7 \%(95 \% \mathrm{Cl} 4.0-5.6)$ and PostOp CR 25.7\%(95\% Cl 23.7-28.2). The observed CRs for all hospitals were under the upper 95\% control limit for both IntraOp and PostOp funnel plots. Risk-adjustment and use of observed-to-expected ratio resulted in one hospital moving to the $>95-98 \% \mathrm{Cl}$ (red) band for IntraOp CRs. Use of only hospital-reported data for PostOp CRs would have resulted in one hospital being unfairly allocated to the red band. There was little concordance between IntraOp and PostOp CRs. 
Conclusions: The funnel plots and overall IntraOp( $\approx 5 \%)$ and PostOp( $\approx 26 \%)$ CRs could be used for benchmarking gynaecological-oncology centres. Hospital benchmarking using risk-adjusted CRs allows fairer institutional comparison. IntraOp and PostOp CRs are best assessed separately. As hospital under-reporting is common for post-operative complications, use of patient-reported outcomes is important.

Keywords: UKGOSOC, complications, gynaecological oncology, risk adjustment, centres, benchmarking, comparison, surgery

Tweetable Abstract: Risk-adjusted benchmarking of surgical complications for ten UK gynaecological oncology centres allows fairer comparison 


\section{Introduction}

There is a drive within the National Health Service (NHS) to increase transparency and improve quality and safety. To this end, one of the initiatives in surgery has been to publish outcomes data for hospitals and more recently for individual surgeons which have been sourced from national clinical audits in some specialties and in most from administrative data. ${ }^{(1,2)}$

While surgical data on a national level has been collected in specialities such as cardiothoracic (3) and orthopaedic (4) surgery and certain cancers such as lung (5), colorectal (6) and head and neck ${ }^{(7)}$, there is paucity of such data in gynaecological oncology (GO). To address this, the United Kingdom Gynaecological Oncology Surgical Outcomes and Complications (UKGOSOC) ${ }^{(8)}$ study was undertaken to prospectively capture data on surgery with a view to setting benchmarking standards. In this cohort, the overall intraoperative (IntraOp) complication rate (CR) was $4.7 \%$ (8) and the postoperative (PostOp) CR was 25.7\%. (9) However use of observed complication rates (CRs) for centre level comparisons does not take into account patient comorbidity, underlying disease or surgical complexity, all of which can impact on the risk of a complication. (8) The use of unadjusted crude CRs has resulted in significant unease amongst surgeons and hospitals due to the variations in prevalence of surgical risk factors. Concerns have been raised that it might deter surgery being undertaken in 'high-risk' patients with significant comorbidity. We report on the impact of risk-adjustment of surgical CRs on benchmarking of GO at hospitals participating in UKGOSOC. 


\section{Methods}

\section{Study Design}

The UKGOSOC study design has been previously described. ${ }^{(8,9)}$ In brief, ten UK GO centres collected data using web-based software on consented women undergoing major gynaecological cancer surgery. Surgeons entered patient co-morbidity, surgical procedures and IntraOp complications (Table S1) contemporaneously in theatre. PostOp complications (Table S2) were defined as occurring up to eight weeks after surgery. These were entered on to the online database during the admission by the hospital team. Following discharge from hospital, patient-reported complications data was also collected using postal follow-up. ${ }^{(9)}$

\section{Statistical Methods}

All reported major surgery was used to calculate IntraOp CR. All reported PostOp complications were graded (I-V) according to severity using the Clavien and Dindo system. ${ }^{(10)}$ Grade 1 complications were excluded from analysis. A PostOp complication for this analysis was defined as one of Grade II-V severity. Only those surgeries where both hospital and patient follow-up data were available, were included in the primary analysis, with PostOp complications including those reported by the clinical team (hospital-reported), the patient (patient-reported) or both. A secondary analysis based on the whole dataset and only hospital-reported PostOp complications, was also undertaken. The outcome was treated as strictly binary and a surgery was coded as having a complication irrespective of the number of complications. ${ }^{(8)}$ The recorded risk factors for IntraOp and PostOp complications have been described previously ${ }^{(8)}$ and are listed in Tables 1 and 3 . Separate comparisons were undertaken for IntraOp and PostOp CRs. All methods described below apply to both.

\section{Data description}


Outcome and categorical risk factors were cross tabulated by hospitals. To assist identification of imbalance in risk factors across hospitals, chi-squared tests were performed. Associated p-values were not used as a formal test measure for the factors with small category counts $(<5)$ at any hospital. Continuous risk factors were summarised by within-hospital means and standard deviations, and $\mathrm{F}$ test statistics and $p$-values from an analysis of variance were similarly used to aid assessment of hospital variation.

\section{Risk prediction and penalised regression}

Logistic regression models were used for risk prediction, though parameter estimates were based on a penalised method (least absolute shrinkage and selection operator or 'lasso') (11) rather than maximum likelihood (ML). A fundamental issue involved in prognostic model construction is that of 'events per variable' (EPV) ${ }^{(12)}$, where the number of 'events' in a binary regression model is taken as the total of the less common outcome. A fitted model should have an EPV of at least $10{ }^{(13,14)}$, where the variable count includes all levels of a categorical variable. The EPV requirement holds even if variable selection (stepwise methods) is performed, so that the variable count is based on the full model. A limited number of EPV can cause validation problems when using $\mathrm{ML}$ for parameter estimates, as the model becomes over-fitted and prediction error is inflated. As a result many prediction models fail to be successfully validated (12).

The lasso deliberately biases (shrinks) the regression estimates toward zero, reducing the mean square prediction error (MSPE) which is a function of the variability, as well as the bias, of the predictions. As a result, despite intentional bias, penalised methods typically provide better prediction than ML. A brief description of the lasso 
method is presented in Appendix 1. Formal inference for biased estimates is dubious so $p$-values should be used only for approximate guidance. ${ }^{(11)}$ The user-written Stata commands plogit and plsearch were used to fit lasso-shrunk logistic models. Equivalent models fitted by $\mathrm{ML}$ are presented for comparison.

Model fit was assessed using the Hosmer-Lemeshow test and model specification with the link test. A receiver operating characteristic $(R O C)$ curve using the predicted probabilities generated by leave-one-out cross-validation (LOO-CV) was calculated and overall performance (discrimination) assessed by the area under the curve (AUC). By regressing the outcome on bootstrapped linear predictions (log odds) for each subject, the calibration slope (CS) ${ }^{(12,13)}$ could be estimated as the mean slope of 1000 bootstrap samples, where a slope close to one suggests good calibration and (much) less than one implies over-fitting of the model. An over-fitted model will give predictions that are too narrow in range.

\section{Hospital rate adjustments}

Observed IntraOp and PostOp CRs for hospitals were compared using funnel plots, a standard approach to institutional comparison. ${ }^{(15,16)}$ They were generated by plotting each hospital's observed CR against sample size and assessed with respect to confidence bands that signify unusually high or low CRs. Control limits for the funnel plots were generated using smoothed exact confidence intervals for the overall CR. These were displayed using coloured 'warning' bands of increasing 'concern' with regard to increasing CRs : green (up to $80 \%)$, yellow ( $\geq 80 \%-90 \%)$, orange ( $\geq 90 \%$ $95 \%)$, and red ( $\geq 95-98 \%)$..

We used the prediction model to produce expected IntraOp and PostOp CRs for each hospital and hence an observed-to-expected CR ratio. Bootstrapped confidence 
intervals for the CR ratio were generated (see Appendix 2) with the same warning levels as for the funnel plots, and if the confidence band contained 1, the hospital was denoted with the appropriate coloured warning.

\section{Results}

\section{Intra-operative complications}

2948 surgeries undertaken across 10 hospitals were included in the analysis. 139 had at least one IntraOp complication. Although the observed IntraOp CR ranged from $2.0 \%$ to $8.0 \%$, the variation was not significant between hospitals $(p=0.052)$.

\section{Modelling and fit}

The distribution of risk factors and CRs across the 10 hospitals is detailed in Table S3. There was variation across hospitals for most predictors, but particularly for laparoscopic approach, grade of surgeon, surgical complexity, final diagnosis and American Society for Anaesthesiologists (ASA) grade.

Of the 2948 surgeries, missing data meant that only 2709 surgeries with 132 complications could be included when fitting the full model. This resulted in an EPV of 4.1 given the 32 variables. The optimised lasso model resulted in four of the 32 variables being excluded. (Table1) Lasso-shrunk odds ratios are presented in Table 1, alongside the ML estimates for comparison. The strongest predictors $(p<0.05$ when estimated by $\mathrm{ML}$ ) of IntraOp complication were surgical complexity (increased risk with increasing complexity), previous abdominal surgery (increased risk), diabetes (increased risk), metabolic-endocrine disorders other than diabetes (decreased risk) and final diagnosis (all cancer types associated with reduced risk relative to ovarian cancer).

The ROC curve based on LOO-CV produced an AUClasso=0.663 (95\%Cl: 0.6160.710), similar to $A \cup C_{M L}=0.659(95 \% \mathrm{Cl}: 0.611-0.706)$, although $\mathrm{ROC}$ curves are 
affected only by rank order and not magnitude. The mean CS lasso of 0.871 suggested a slight narrowness of predictions, although the $2.5^{\text {th }}-97.5^{\text {th }}$ centile of the slopes (0.717-1.068) contain the optimal value of 1 . In contrast, the $\mathrm{CS}_{\mathrm{ML}}=0.712(95 \% \mathrm{Cl}$ : 0.364-0.887) indicated that the prediction range was limited, and hence the model over-fitted, without parameter shrinkage.

\section{Hospital rate adjustments}

Figure 1a shows the funnel plot allowing a simple comparison of observed IntraOp CR by hospital. A majority of the hospitals are within the green band with some, such as Hospital F, having a significantly low IntraOp CR outside the 95\% ( $\approx 2$ standard deviations) control limits. Hospitals $\mathrm{J}$ and $\mathrm{E}$ have $\mathrm{CR}$ higher than the overall IntraOp CR but the moderate number of surgeries analysed from these hospitals (150 and 181 , respectively) means a reduced confidence in their outlier status and they lie within the yellow band (control limits $\geq 80 \%-90 \%$ ). Figure $1 \mathrm{~b}$ shows the observed-to-expected CR ratio, adjusted for risk factor prevalence in the 10 hospitals. The spread of expected IntraOp CR for hospitals is between $3.9 \%$ and $5.4 \%$ (Table $2 \mathrm{a}$ ). In Figure $1 \mathrm{~b}$ Hospital $F$ is confirmed as having a low IntraOp CR after adjustment. The 95\% confidence interval for Hospital $E$ is completely above 1, the line of equality (observed=expected), and it is coded red (confidence interval $>95-98 \%$ ) marking it as a centre with high IntraOp CR. The ratio for Hospital $\mathrm{G}$ is also high at 1.8, but with wide confidence intervals, hence it remains coded green. Hospital J, which had the highest observed IntraOp CR, only has the $3^{\text {rd }}$ highest ratio (Table $2 \mathrm{a}$ ) and remains coded yellow, indicating that its high $\mathrm{CR}$ is partially mitigated by a relatively high-risk case-mix of surgeries. 


\section{Post-operative complications}

The primary PostOp CR analysis was restricted to the subset of 1462 surgeries with both hospital and patient reported outcomes. 376 had at least one PostOp complication. Individual hospital statistics are presented in Table S4. Estimated blood loss (EBL) and duration of surgery varied significantly by hospital. The PostOp CR varied from $15.6 \%$ to $36.2 \%$ between hospitals but the difference was not significant $(p=0.096)$. The findings were similar for the full dataset (Table S3).

\section{Modelling and fit}

Of the 1462 surgeries missing data meant only 1371 surgeries with 346 events were included when fitting the full model. This resulted in an EPV of 9.9 given the 35 variables. The optimised lasso model resulted in 15 variables out of 35 being removed from the model (Table 3). Only duration of surgery appeared to be a strong predictor of PostOp complications, though coagulation-thrombosis, and musculoskeletal disorders and diabetes (all increase risk), laparoscopic approach (decreases risk) and final diagnosis (cervical and vulval cancer increase risk relative to ovarian cancer) were significant at the $5 \%$ level using ML. The ROC curve based on LOO-CV produced an $\mathrm{AUC}_{\text {lasso }}=0.659(95 \% \mathrm{Cl}: 0.585-0.733)$, significantly larger than $\mathrm{AUC} \mathrm{ML}_{\mathrm{L}}=0.569$ (95\% Cl:0.487-0.652) as tested using the method described by DeLong et al $(17)(p=0.0003)$. The mean $C_{\text {lasso }}=1.008(95 \% \mathrm{Cl}: 0.799-1.264)$ suggested nearperfectly calibrated predictions. By comparison, a mean CSML $=0.689(95 \% \mathrm{Cl}$ : $0.562-$ 0.835) strongly indicated that the ML-based prediction range was too narrow, and hence the model was over-fitted.

\section{Hospital rate adjustments}


Figure 1c compares the observed PostOp CRs of the 10 hospitals using a funnel plot. The overall CR was $25.7 \%$. None of the hospitals had a PostOp CR that was significantly higher than the overall CR. Hospital J had the highest PostOp CR (36.2\%) but as this was based on only 58 surgeries, it lies within the yellow (control limits $\geq 80 \%$ 90\%) band. Hospitals G (15.6\%) and I (17.1\%) have notably low CR. Figure 1d shows the observed-to-expected PostOp CR ratio, with actual values found in Table $2 b$. The spread of expected PostOp CR for hospitals is between $20.1 \%$ and $28.5 \%$. None of the hospitals have a $C R$ ratio significantly different above 1 , the line of equality (observed=expected) at the $5 \%$ level, though Hospital $\mathrm{J}$ remains within the yellow band.

All PostOp CRs are lower if hospital reported statistics alone are used. (Figure S1faded colour-coding) Hospital $\mathrm{D}$ is in the red band when hospital reported rates alone are used. However, when surgeries with both hospital and patient reported statistics are used, hospital $D$ is in the green band suggesting that differences in under-reporting can significantly impact on hospital comparisons. (Figure S1 - bright colour-coding)

Generally, there was little concordance between IntraOp and PostOp CR. Hospitals G and $E$ which were among the centres with high IntraOp CR, had some of the lowest PostOp CRs, both observed and risk-adjusted. (Figure 1)

\section{Discussion}

\section{Main Findings}

Risk-adjustment did not make a significant difference to the CRs for majority of centres, but helped to delineate the outliers better. The shaded funnel plots and observed versus expected ratios generated made comparisons easy to comprehend. 
Hospital under-reporting is common for PostOp complications and inclusion of patientreported outcomes is important to ensure valid comparison between institutions. Risk factors for IntraOp and PostOp CRs were largely different and even after adjustment there was no concordance between hospital IntraOp and PostOp CRs. The overall IntraOp $(\approx 5 \%)$ and PostOp $(\approx 26 \%)$ CR derived from this study could be used to benchmark performance in GO.

\section{Strength and Limitations}

This is the first large prospective multicentre study in GO to develop risk-adjusted CRs for inter-institutional comparison of surgical outcomes. Although such data is available in other specialties, $(3,5,7,18)$ in $\mathrm{GO}$, it is limited to a retrospective study comparing outcomes of ovarian cancer surgery between three US tertiary centres. ${ }^{(19)}$

Whilst the limited number of surgeries entered is not entirely representative of all GO operations performed in the UK, this was a huge undertaking for the clinicians involved. For the 7 centres we have data for, the inclusion rate of cases ranged from $64.6 \%$ to $97.6 \%$ and all cases were prospectively registered prior to surgery.

We have previously described the risk predictors for IntraOp and PostOp CRs based on univariable and multivariable regression. ${ }^{\left({ }^{8}\right)}$ Few of the factors appeared important across either model reflecting the difficulty in developing risk-prediction tools. While significant effort was required for complete prospective data collection on 2948 surgeries, given the high EPV rate had significant implications for estimation.

Use of a data-dependent internal measure (observed overall $\mathrm{CR}$ ) in lieu of a prespecified target rate based on external data and expert opinion was a limitation, and 
a hospital with a particularly high rate will help push up that value to which all hospitals are compared to. Unfortunately there was insufficient published data on GO CRs to utilise a prior target rate. Given that there are no apparent institutional outliers in our dataset, the observed CRs might be reasonably used as future target rates. A related issue is that the data used to estimate the prediction model was the same to which the model was then applied, though cross-validation methods were employed. An external dataset is therefore required for proper model validation.

\section{Interpretation}

Figure 1 suggests the adjustment process may appear to add little value when comparing centres, given that most of the predictors appeared to have limited impact on outcome. However adjustment helps to better define the level of excess surgical complications at a given hospital, and could therefore provide an earlier intimation of potential issues. Hospital E was only flagged as having a statistically high IntraOp CR $(p=0.03)$ following adjustment. The IntraOp and PostOp CRs did not vary significantly between the 10 hospitals, so that for the majority, the observed CRs were within the funnel plot control limits. In the broader healthcare community, where the spread of quality and CRs is likely to be wider, it is likely that there will be institutions beyond the various safety bounds, requiring more precise performance monitoring.

By contrast, nearly all the predictors varied considerably by hospital, especially those involving an element of surgical decision making (laparoscopic approach, surgeon grade, surgical complexity). This 'inter-hospital' variability in risk factor prevalence is a strong argument in itself for the need to adjust for fairer comparison. That riskadjustment did not substantively affect results is partially due to parameter shrinkage caused by lack of statistical power. Based on our results, a subsequent study 
modelling IntraOp CRs, would need $n \approx 12000$ for hypertension $\left(O R_{M L}=1.28 ; p=0.26\right)$ to achieve power $=80 \%$, for example. With more data some of these factors may contribute significantly to CR prediction, both statistically and clinically. The lack of association between $\mathrm{CR}$ and factors like BMI, especially after open surgery, are contrary to previous reports. $(20,21)$ However, it is evident that much of the outcome variability is related to unmeasured (perhaps unobservable) phenomena, and we do not expect a surgical complication to be ever predicted with a high degree of confidence.

The difference in ranking order of hospitals for IntraOp and PostOp CRs, for example, hospitals $G$ and $E$, could be due to various reasons including surgical skill, postoperative care and under-reporting of PostOp complications. Also, since IntraOp and PostOp CRs had differing risk factors, we recommend assessing hospitals separately for IntraOp and PostOp complications as combining them could mask deficiencies in perioperative care at certain hospitals.

Analysis of only hospital-reported PostOp complications demonstrated that hospital D had the highest CR outside the control limits (Figure S1). However, with inclusion of patient-reported data hospital D was no longer an outlier, but hospital J's CR increased from close to the $50^{\text {th }}$ to the $90^{\text {th }}$ centile, suggesting hospital $D$ had been more diligent at recording all PostOp complications. These findings further substantiate the need for including patient-reported PostOp complications to overcome clinician underreporting.

Penalized models may appear complicated but in a limited event situation it is known that selection methods may moderate predictors and include noise predictors. (22) However, it is straightforward to input predictor values into, say, an Excel sheet preprepared with the necessary formula to calculate risk scores, and calculate confidence 
limits treating the expected rate as fixed (23) (Table S5). Alternatively, the model parameters presented could be used as informative priors for subsequent model building by other researchers in a Bayesian context.

Since morbidity is the main yardstick for benchmarking surgical performance, moving forwards, it would be important to have complete and accurate data in a larger database. The drawback of clinician-led databases is that all surgical episodes may not get recorded due to its heavy reliance on voluntary data entry. ${ }^{(24)}$ Also, some centres with high morbidity rates may be hesitant to enter all their data. ${ }^{(25)}$ The alternative would be an administrative database like Hospital Episode Statistics (HES) where all surgical episodes are automatically recorded, which, might however have incomplete complications data. Although Nouraei et al ${ }^{(7)}$ found CRs derived from HES comparable to the clinician-led head and neck surgery database, this has not been the case with other specialties. ${ }^{(26)} \mathrm{A}$ re-audit in one of the participating centres demonstrated higher CRs than that derived from HES, but comparable to UKGOSOC.

(27) Therefore, a reasonable compromise may be to combine HES with a clinician-led database. To ensure completeness it would be prudent to minimise the data fields requiring clinician entry. It is hoped that mandatory requirement to publish individual surgeon's outcomes will encourage complete and accurate data entry. ${ }^{(1)}$ Valid casemix risk-adjustment is also likely to reassure surgeons and encourage active participation in surgical outcomes assessment audits.

\section{Conclusion}

Risk-adjustment had a modest effect on the rankings of the individual centres based on their CRs. However, by accounting for the prevalence of potential risk factors we may be able to estimate an adjusted $\mathrm{CR}$ that ensures fairer inter-institutional 
comparison. The overall IntraOp $(\approx 5 \%)$ and PostOp $(\approx 26 \%)$ CRs and funnel plots could be used to benchmark performance of GO centres and even individual surgeons with a larger dataset. The risk factors for IntraOp and PostOp complications are different and it is important to report on the two CRs separately.

\section{Disclosure of interests}

The authors have no conflicts of interest to declare.

\section{Contribution to authorship}

UM was the principal investigator of the study. UM, AN, AGM, were responsible for conceptualisation and design of the study. RL, AGM, RI, AN and UM designed the database. RM, ND, RG, ABG, KH, SL, An L, AI L, TM, JN, AO, BR, SS, NW, KR were involved with recruitment and data entry from individual centres. AT and DM helped with hosting the database on the cancer-registry server, issuing passwords for all users and sending patient follow-up letters. RI was responsible for cleaning and preparing the data for the analysis, analysing patient follow-up questionnaires and writing of the manuscript with MB. MB performed the statistical analysis and drafted the manuscript. AR helped with cleaning of the data. All the authors provided regular feedback on the manuscript.

\section{Acknowledgements}

This study was supported by researchers at the National Institute for Health Research, University College London Hospitals Biomedical Research Centre. The authors are thankful to the Eve appeal for their support of the study. The authors are also very grateful to all the women who participated and to all the medical, nursing and 
administrative staff who worked on UKGOSOC. In addition the authors would like to thank the following members of the hospital teams who contributed to data collection: Sara Roberts, Philip Toon, Richard Peevor (Betsi Cadwaladr University Health Board), Charlie Chan, Janos Balega, Kavita Singh, Sudha Sundar, Ahmed Elattar, Esther Moss, Mary Wright (City Hospital Birmingham), Alta Viljoen (Cheltenham General Hospital), Branislav Potancok, Mohamed Ismail, Vivek Nama, Ruth Lomas, Cheryl Walke (East Kent University Hospital NHS Foundation Trust), Deborah Woods, Alison Garnham (Ipswich General Hospital), KetanGajjar, Deborah Parkinson (Royal Preston Hospital), Emma Arthur, Arnold Kruse (Royal Cornwall Hospital), Kostas Doufekas (University College London Hospital). The study was supported by researchers at the National Institute for Health Research University College London Hospitals Biomedical Research Centre.

\section{Ethics Approval}

The joint UCL and UCLH committees decided that this study did not require ethics approval. However consent was taken from the women who participated in the study to include their identifiers so that they could be sent a follow-up letter following their discharge from hospital.

\section{Funding}

We are grateful to the Eve Appeal for the funding of this study. 


\section{References}

1. http://www.nhs.uk/service-search/performance/Consultants\#view-the-data.

2. http://www.hqip.org.uk/consultant-outcomes-publication/.

3. Bridgewater B. Cardiac registers: the adult cardiac surgery register. Heart. 2010 September 1, 2010;96(18):1441-3.

4. Hunt LP, Ben-Shlomo Y, Clark EM, Dieppe P, Judge A, MacGregor AJ, et al. 90-day mortality after 409096 total hip replacements for osteoarthritis, from the National Joint Registry for England and Wales: a retrospective analysis. The Lancet. //28;382(9898):1097-104.

5. Beckett Pcp, Woolhouse Icp, Stanley R, Peake MDcp. Exploring variations in lung cancer care across the UK - the 'story so far' for the National Lung Cancer Audit. Clinical medicine. 2012;12(1):14-8.

6. The National Bowel Cancer Audit Report 2010 http://www.ic.nhs.uk/webfiles/Services/NCASP/audits\%20and\%20reports/NHS Bowel_Cancer 2010 INTERACTIVE.pdf. 2010. 
7. Nouraei SAR, Middleton SE, Hudovsky A, Darzi A, Stewart S, Kaddour H, et al. A national analysis of the outcome of major head and neck cancer surgery: implications for surgeon-level data publication. Clinical otolaryngology \& allied sciences. 2013;38(6):502-11.

8. Iyer R, Gentry-Maharaj A, Nordin A, Burnell M, Liston R, Manchanda R, et al. Predictors of complications in gynaecological oncological surgery: a prospective multicentre study (UKGOSOC[mdash]UK gynaecological oncology surgical outcomes and complications). $\mathrm{Br} \mathrm{J}$ Cancer. 2014 12/23/online.

9. Iyer R, Gentry Maharaj A, Nordin A, Liston R, Burnell M, Das N, et al. Patient-reporting improves estimates of postoperative complication rates: A prospective cohort study in gynaecological oncology. British Journal of Cancer. 2013;109(3):623.

10. Dindo D, Demartines N, Clavien PA. Classification of surgical complications - A new proposal with evaluation in a cohort of 6336 patients and results of a survey. Annals of surgery. 2004;240(2):205-13.

11. Tibshirani R. Regression Shrinkage and Selection via the Lasso. Journal of the Royal Statistical Society Series B (Methodological). 1996;58(1):267-88.

12. Ambler G, Seaman S, Omar RZ. An evaluation of penalised survival methods for developing prognostic models with rare events. Statistics in medicine. 2012 May 20;31(1112):1150-61. PubMed PMID: 21997569.

13. Ambler G, Brady AR, Royston P. Simplifying a prognostic model: a simulation study based on clinical data. Statistics in medicine. 2002 Dec 30;21(24):3803-22. PubMed PMID: 12483768.

14. Moons KGM, Kengne AP, Woodward M, Royston P, Vergouwe Y, Altman DG, et al. Risk prediction models: I. Development, internal validation, and assessing the incremental value of a new (bio)marker. Heart. 2012 May;98(9):683-90. PubMed PMID: WOS:000303859300004. English.

15. Spiegelhalter $D$. Funnel plots for institutional comparison. Quality \& safety in health care. 2002 Dec;11(4):390-1. PubMed PMID: 12468705. Pubmed Central PMCID: 1757996.

16. Spiegelhalter DJ. Funnel plots for comparing institutional performance. Statistics in medicine. 2005 Apr 30;24(8):1185-202. PubMed PMID: 15568194.

17. Delong ER, Delong DM, Clarkepearson DI. Comparing the Areas under 2 or More Correlated Receiver Operating Characteristic Curves - a Nonparametric Approach. Biometrics. 1988 Sep;44(3):837-45. PubMed PMID: WOS:A1988Q069100016. English.

18. National Bowel Cancer Audit Programme : Healthcare Quality Improvement Partnership (HQIP). http://www.hqip.org.uk/national-bowel-cancer-audit-programme-nbocap.Reports of the National Bowel Cancer Audit Project 2002-2006. .

19. Aletti GD, Santillan A, Eisenhauer EL, Hu J, Aletti G, Podratz KC, et al. A new frontier for quality of care in gynecologic oncology surgery: Multi-institutional assessment of short-term outcomes for ovarian cancer using a risk-adjusted model. Gynecologic Oncology. 2007;107(1):99-106. 
20. Kumar A, Bakkum-Gamez JN, Weaver AL, McGree ME, Cliby WA. Impact of obesity on surgical and oncologic outcomes in ovarian cancer. Gynecologic Oncology. 2014 10//;135(1):1924.

21. Osler M, Daugbjerg S, Frederiksen B, Ottesen B. Body mass and risk of complications after hysterectomy on benign indications. Human reproduction.26(6):1512-8.

22. Royston P, Sauerbrei W. Multivariable Model-Building: A Pragmatic Approach to Regression Analysis Based on Fractional Polynomials for Modelling Continuous Variables. Wiley: Chichester, UK. 2008.

23. DeLong ER, Peterson ED, DeLong DM, Muhlbaier LH, Hackett S, Mark DB. Comparing risk-adjustment methods for provider profiling. Statistics in Medicine. 1997;16(23):2645-64.

24. RCOG. Hospital Episode Statistics as a source of information on safety and quality in gynaecology to support revalidation. 2012.

25. Almoudaris AM, Burns EM, Bottle A, Aylin P, Darzi A, Faiz O. A colorectal perspective on voluntary submission of outcome data to clinical registries. British Journal of Surgery. 2011;98(1):132-9.

26. Cockbain AJ, Carolan M, Berridge D, Toogood GJ. Performance and Quality Indicators: The importance of accurate coding. Bulletin of the Royal College of Surgeons of England. 2012;94(2):46-50.

27. Rajkumar S, Lee MJ, Nordin A. Perioperative morbidity and mortality audit strategies in gynaecological oncology surgery: The east Kent gynaecological oncology centre experience. International journal of gynecological cancer. 2014;24(9 SUPPL. 4):1160-1. 


\section{Appendix S1: The Lasso method}

The lasso (least absolute shrinkage and selection operator) estimator [1] employs a penalty term in the likelihood function that is then maximised subject to a constraint on the (absolute) sum of the regression coefficients. The penalty term is a function of shrinkage parameter $(\lambda)$ chosen by the investigator, which when equal to zero reduces to ML estimation and when tending to infinity results in estimates tending to zero. In contrast to the similar ridge regression method, where all the coefficients of the full model are partially shrunk, the lasso actually performs a type of variable selection. Strong and moderate predictors are shrunk by a certain amount dependent on $\lambda$, whilst weak predictors may be shrunk to exactly zero and so drop out of the model. The choice of $\lambda$ here was based on a grid search that minimised the generalized crossvalidation error [1]. Note that inference, such as confidence intervals and $p$-values, based on standard errors from the lasso variance-covariance matrix should be treated with caution and used only for approximate guidance. Standard errors are not particularly meaningful for (deliberately and quite strongly) biased estimates as they will exclude the inaccuracy caused by bias [1].

In contrast to penalized regression methods, model selection procedures are known to result in selection- or omission-bias [2], whereby weakly significant variables will be infrequently selected, dependent on chance variation, and when selected, they will typically have overestimated coefficients.

Appendix S2: The observed-expected complication rate and confidence intervals 
We used the risk prediction model to produce expected IntraOp and PostOp CRs for each hospital by summing the predicted risk for each surgery over each hospital. We then compared the expected with the observed $\mathrm{CR}$, and if the confidence band for the $\mathrm{CR}$ ratio (defined as for the funnel plots: green (up to $80 \%$ ), yellow ( $\geq 80 \%-90 \%$ ), orange ( $\geq 90 \%-95 \%)$, and red ( $\geq 95-98 \%))$ contained 1 , we denoted the hospital with the appropriate coloured warning. To incorporate the uncertainty involved in estimating the expected $C R$, as well as the choice of $\lambda$, the sampling distribution of the observedto-expected CR ratio was estimated by taking 1000 bootstrap samples of the full dataset. For each bootstrap sample the new observed-to-expected CR ratio was calculated for each hospital, based on a refitting of the lasso model with $\lambda$ optimally selected. Confidence intervals for the ratio were based on the appropriate biascorrected centiles of the bootstrap-derived sampling distribution.

\section{References:}

1. Tibshirani R. Regression shrinkage and selection via the Lasso. J Roy Stat Soc B Met 1996;58(1):267-88

2. Royston P, Sauerbrei W. Multivariable Model-Building: A Pragmatic Approach to Regression Analysis Based on Fractional Polynomials for Modelling Continuous Variables. Wiley: Chichester, UK 2008 
Figure 1: Comparison of intraoperative ( $a$ and $b$ ) and patient reported postoperative ( $c$ and $d$ ) complication rates. Left hand panels plot observed rates over colour-coded funnel plots where the yellow band/line represents a $80-90 \%$ control limit, the orange band/line a 90-95\% control limit, and the red band/line a 95-98\% control limit around overall rate. Right hand panels plot ratio of observed to expected rate against the null value of one, with colour-coded confidence intervals representing the same interval range as for the funnel plots. All plotted rates are also colour-coded, reflecting the position of the observed rate (left panels) or null value (right panels) within the appropriate coloured band.

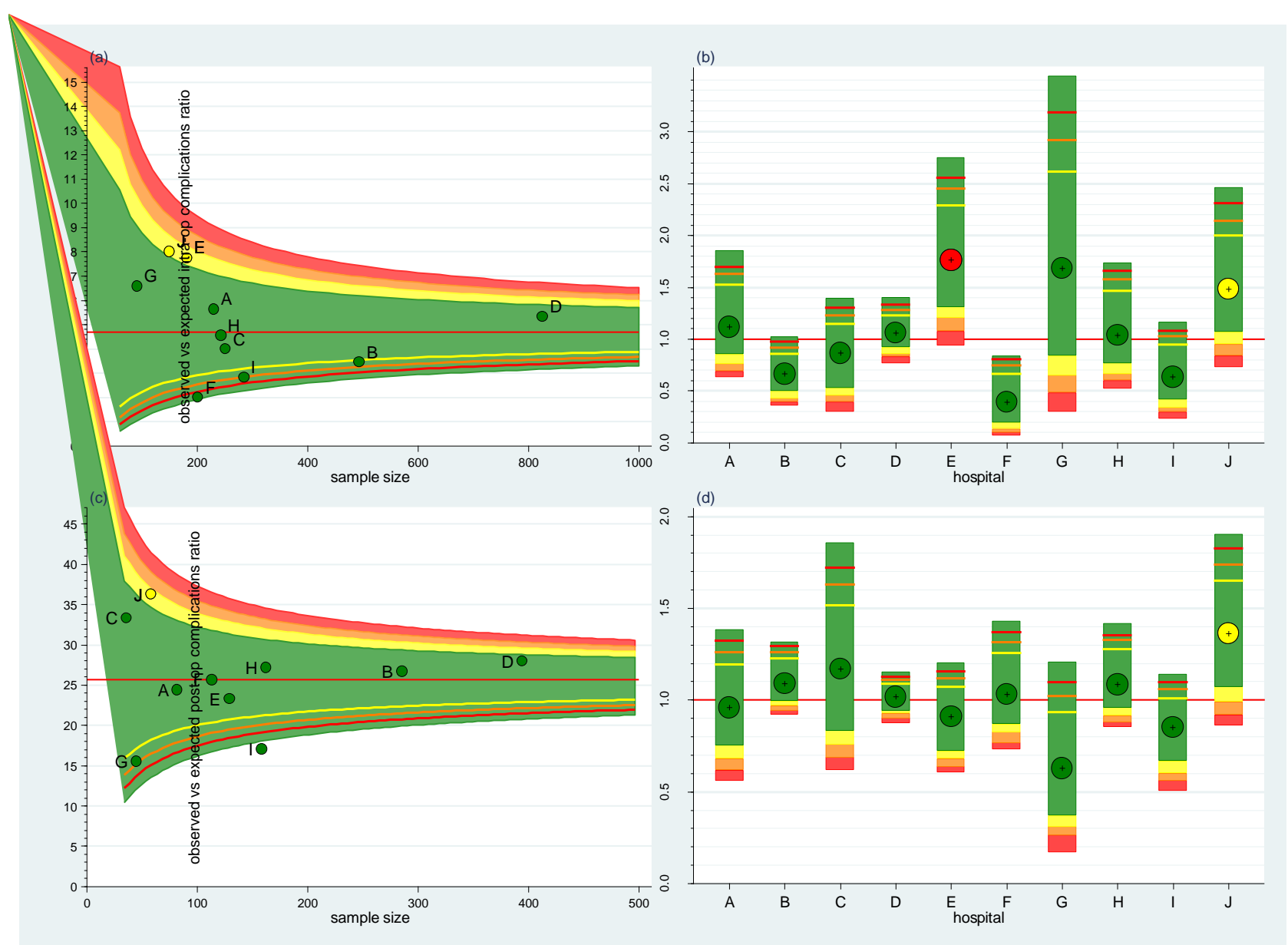


Table 1: Risk prediction model for intra-operative complications - full sample of hospital reported outcomes $n=2948$

\begin{tabular}{|c|c|c|c|c|c|c|c|c|c|}
\hline \multirow{2}{*}{ Variables } & \multicolumn{4}{|c|}{ Lasso } & \multicolumn{4}{|c|}{ Maximum Likelihood } & \multirow{2}{*}{ Shrinkage } \\
\hline & $\mathrm{OR}$ & $\mathrm{p}$-value ${ }^{*}$ & $\mathrm{~L} 95 \% \mathrm{Cl}^{*}$ & U95\% $\mathrm{Cl}^{*}$ & OR & p-value & $\mathrm{L} 95 \% \mathrm{Cl}$ & $\mathrm{U} 95 \% \mathrm{Cl}$ & \\
\hline Age at surgery & 1.000 & 0.977 & 0.985 & 1.015 & 1.001 & 0.859 & 0.986 & 1.017 & $-84.0 \%$ \\
\hline BMI & removed & & & & 1.003 & 0.867 & 0.974 & 1.032 & $-100.0 \%$ \\
\hline Previous abdominal surgery & 1.426 & 0.058 & 0.988 & 2.057 & 1.459 & 0.045 & 1.008 & 2.111 & $-6.0 \%$ \\
\hline Low albumin & 3.916 & 0.118 & 0.709 & 21.645 & 4.461 & 0.080 & 0.836 & 23.799 & $-8.7 \%$ \\
\hline Coagulation-thrombosis & 1.052 & 0.910 & 0.436 & 2.540 & 1.148 & 0.755 & 0.483 & 2.729 & $-63.1 \%$ \\
\hline Diabetes & 1.804 & 0.032 & 1.052 & 3.095 & 1.923 & 0.018 & 1.118 & 3.306 & $-9.7 \%$ \\
\hline Cardiac & 1.462 & 0.205 & 0.812 & 2.632 & 1.572 & 0.128 & 0.878 & 2.814 & $-15.9 \%$ \\
\hline Respiratory & 0.676 & 0.266 & 0.339 & 1.348 & 0.573 & 0.133 & 0.277 & 1.185 & $-29.8 \%$ \\
\hline Gastrointestinal & 1.065 & 0.893 & 0.425 & 2.668 & 1.188 & 0.703 & 0.490 & 2.879 & $-63.5 \%$ \\
\hline Genitourinary & 0.699 & 0.679 & 0.129 & 3.805 & 0.486 & 0.483 & 0.065 & 3.651 & $-50.4 \%$ \\
\hline Musculoskeletal & removed & & & & 1.091 & 0.791 & 0.574 & 2.071 & $-100.0 \%$ \\
\hline Neurology-psychiatric & removed & & & & 1.028 & 0.940 & 0.501 & 2.109 & $-100.0 \%$ \\
\hline Vascular & 0.849 & 0.775 & 0.276 & 2.607 & 0.675 & 0.527 & 0.199 & 2.286 & $-58.3 \%$ \\
\hline Auto-immune & 1.968 & 0.253 & 0.617 & 6.282 & 2.132 & 0.191 & 0.685 & 6.642 & $-10.6 \%$ \\
\hline Metabolic-endocrine & 0.412 & 0.027 & 0.187 & 0.906 & 0.329 & 0.010 & 0.141 & 0.768 & $-20.3 \%$ \\
\hline Integumentary-dermatology & removed & & & & 0.964 & 0.965 & 0.191 & 4.873 & $-100.0 \%$ \\
\hline Hypertension & 1.239 & 0.325 & 0.808 & 1.899 & 1.279 & 0.263 & 0.831 & 1.969 & $-13.0 \%$ \\
\hline Smoking & 0.978 & 0.969 & 0.323 & 2.961 & 0.828 & 0.760 & 0.246 & 2.788 & $-88.2 \%$ \\
\hline Other neoplasms & 1.506 & 0.246 & 0.755 & 3.004 & 1.590 & 0.182 & 0.805 & 3.140 & $-11.8 \%$ \\
\hline Laparoscopic approach & 1.021 & 0.935 & 0.618 & 1.689 & 1.240 & 0.403 & 0.749 & 2.051 & $-90.2 \%$ \\
\hline \multicolumn{10}{|l|}{ ASA } \\
\hline ASA grade 1 & 1 & & & & 1 & & & & \\
\hline ASA grade 2 & 1.103 & 0.699 & 0.670 & 1.816 & 1.250 & 0.401 & 0.742 & 2.106 & $-56.0 \%$ \\
\hline ASA grade $3+$ & 1.039 & 0.908 & 0.539 & 2.003 & 1.183 & 0.628 & 0.599 & 2.336 & $-77.0 \%$ \\
\hline
\end{tabular}




\begin{tabular}{|c|c|c|c|c|c|c|c|c|c|}
\hline Consultant & 1 & & & & 1 & & & & \\
\hline Sub-specialty trainee & 0.716 & 0.604 & 0.202 & 2.535 & 0.614 & 0.460 & 0.168 & 2.243 & $-31.6 \%$ \\
\hline General Obstetrics \& Gynaecology Trainee & 1.286 & 0.673 & 0.399 & 4.144 & 1.243 & 0.720 & 0.378 & 4.083 & $15.8 \%$ \\
\hline \multicolumn{10}{|l|}{ Surgical complexity } \\
\hline Complexity score $1 \& 2$ & 1 & & & & 1 & & & & \\
\hline Complexity score $3 \& 4$ & 1.097 & 0.692 & 0.695 & 1.731 & 1.263 & 0.325 & 0.794 & 2.009 & $-60.4 \%$ \\
\hline Complexity score $5 \& 6$ & 1.905 & 0.016 & 1.130 & 3.212 & 2.208 & 0.003 & 1.298 & 3.756 & $-18.6 \%$ \\
\hline Complexity score $7 \& 8$ & 2.666 & 0.012 & 1.242 & 5.725 & 3.080 & 0.004 & 1.434 & 6.612 & $-12.8 \%$ \\
\hline Complexity score $>8$ & 4.005 & 0.003 & 1.626 & 9.865 & 4.561 & 0.001 & 1.850 & 11.242 & $-8.6 \%$ \\
\hline \multicolumn{10}{|l|}{ Final diagnosis } \\
\hline Ovarian & 1 & & & & 1 & & & & \\
\hline Uterine & 0.600 & 0.050 & 0.360 & 1.001 & 0.504 & 0.011 & 0.296 & 0.856 & $-25.5 \%$ \\
\hline Cervical & 0.834 & 0.636 & 0.393 & 1.769 & 0.696 & 0.361 & 0.320 & 1.514 & $-49.9 \%$ \\
\hline Vulval & 0.289 & 0.049 & 0.084 & 0.993 & 0.195 & 0.026 & 0.046 & 0.826 & $-24.1 \%$ \\
\hline Benign & 0.567 & 0.041 & 0.329 & 0.976 & 0.508 & 0.017 & 0.291 & 0.887 & $-16.2 \%$ \\
\hline Constant & 0.033 & 0.000 & 0.007 & 0.146 & 0.025 & 0.000 & 0.004 & 0.145 & $-32.8 \%$ \\
\hline
\end{tabular}

* for approximate guidance only 
Table 2: Summary of intraoperative and postoperative complications by centre

\begin{tabular}{|c|c|c|c|c|c|c|c|c|}
\hline \multicolumn{9}{|c|}{ a) Intraoperative complications summary - full sample of hospital reported outcomes n=2948 } \\
\hline Hospital & $\begin{array}{l}\text { Number of } \\
\text { surgeries }\end{array}$ & $\begin{array}{l}\text { Number } 10^{1} \\
\text { complications }\end{array}$ & $\begin{array}{c}\text { Observed IO } \\
\mathrm{CR}^{2}\end{array}$ & $\begin{array}{c}\text { Expected } \\
\text { number } 10 \\
\text { complications }\end{array}$ & $\begin{array}{c}\text { Expected } 10 \\
\text { CR }\end{array}$ & $\begin{array}{l}\text { O/E } E^{3} \text { IO CR } \\
\quad \text { ratio }\end{array}$ & $\begin{array}{l}\text { Lower } 95 \% \mathrm{Cl}^{4} \\
\text { for O/E ratio }\end{array}$ & $\begin{array}{l}\text { Upper } 95 \% \mathrm{Cl} \\
\text { for O/E ratio }\end{array}$ \\
\hline A & 230 & 13 & $5.7 \%$ & 11.7 & $5.1 \%$ & 1.116 & 0.689 & 1.698 \\
\hline B & 493 & 17 & $3.4 \%$ & 25.6 & $5.2 \%$ & 0.664 & 0.390 & 0.977 \\
\hline C & 251 & 10 & $4.0 \%$ & 11.6 & $4.6 \%$ & 0.865 & 0.389 & 1.307 \\
\hline$D$ & 825 & 44 & $5.3 \%$ & 41.7 & $5.1 \%$ & 1.055 & 0.826 & 1.333 \\
\hline$E$ & 181 & 14 & $7.7 \%$ & 8.0 & $4.4 \%$ & 1.761 & 1.072 & 2.554 \\
\hline $\mathbf{F}$ & 201 & 4 & $2.0 \%$ & 10.2 & $5.1 \%$ & 0.393 & 0.102 & 0.804 \\
\hline $\mathbf{G}$ & 91 & 6 & $6.6 \%$ & 3.6 & $3.9 \%$ & 1.681 & 0.481 & 3.189 \\
\hline $\mathrm{H}$ & 242 & 11 & $4.5 \%$ & 10.6 & $4.4 \%$ & 1.035 & 0.598 & 1.659 \\
\hline 1 & 284 & 8 & $2.8 \%$ & 12.6 & $4.4 \%$ & 0.635 & 0.298 & 1.081 \\
\hline $\mathrm{J}$ & 150 & 12 & $8.0 \%$ & 8.1 & $5.4 \%$ & 1.479 & 0.838 & 2.313 \\
\hline \multicolumn{9}{|c|}{ b) Postoperative complications summary - sample restricted to hospital and patient reported outcomes $n=1462$} \\
\hline Hospital & $\begin{array}{l}\text { Number of } \\
\text { surgeries }\end{array}$ & $\begin{array}{l}\text { Number PO5 } \\
\text { complications }\end{array}$ & $\begin{array}{c}\text { Observed PO } \\
\text { CR }\end{array}$ & $\begin{array}{c}\text { Expected } \\
\text { number PO } \\
\text { complications }\end{array}$ & $\begin{array}{c}\text { Expected PO } \\
\text { CR }\end{array}$ & $\begin{array}{l}\text { O/E PO CR } \\
\text { ratio }\end{array}$ & $\begin{array}{l}\text { Lower } 95 \% \mathrm{Cl} \\
\text { for O/E ratio }\end{array}$ & $\begin{array}{l}\text { Upper } 95 \% \mathrm{Cl} \\
\text { for O/E ratio }\end{array}$ \\
\hline $\mathrm{A}$ & 82 & 20 & $24.4 \%$ & 20.9 & $25.4 \%$ & 0.958 & 0.616 & 1.325 \\
\hline B & 285 & 76 & $26.7 \%$ & 69.7 & $24.4 \%$ & 1.091 & 0.942 & 1.296 \\
\hline$C$ & 36 & 12 & $33.3 \%$ & 10.3 & $28.5 \%$ & 1.171 & 0.688 & 1.723 \\
\hline D & 394 & 110 & $27.9 \%$ & 108.2 & $27.5 \%$ & 1.017 & 0.902 & 1.128 \\
\hline$E$ & 129 & 30 & $23.3 \%$ & 33.0 & $25.5 \%$ & 0.910 & 0.638 & 1.157 \\
\hline $\mathbf{F}$ & 113 & 29 & $25.7 \%$ & 28.2 & $24.9 \%$ & 1.029 & 0.769 & 1.370 \\
\hline
\end{tabular}




\begin{tabular}{|l|l|l|l|l|l|l|l|l|}
$\mathbf{G}$ & 45 & 7 & $15.6 \%$ & 11.1 & $24.8 \%$ & 0.628 & 0.265 & 1.098 \\
\hline $\mathbf{H}$ & 162 & 44 & $27.2 \%$ & 40.5 & $25.0 \%$ & 1.086 & 0.881 & 1.355 \\
\hline $\mathbf{I}$ & 158 & 27 & $17.1 \%$ & 31.8 & $20.1 \%$ & 0.850 & 0.562 & 1.099 \\
\hline $\mathbf{J}$ & 58 & 21 & $36.2 \%$ & 15.4 & $26.6 \%$ & 1.361 & 0.917 & 1.828 \\
\hline
\end{tabular}


Table 3: Risk prediction model for Postoperative complications - sample restricted to hospital and patient reported outcomes $n=1462$

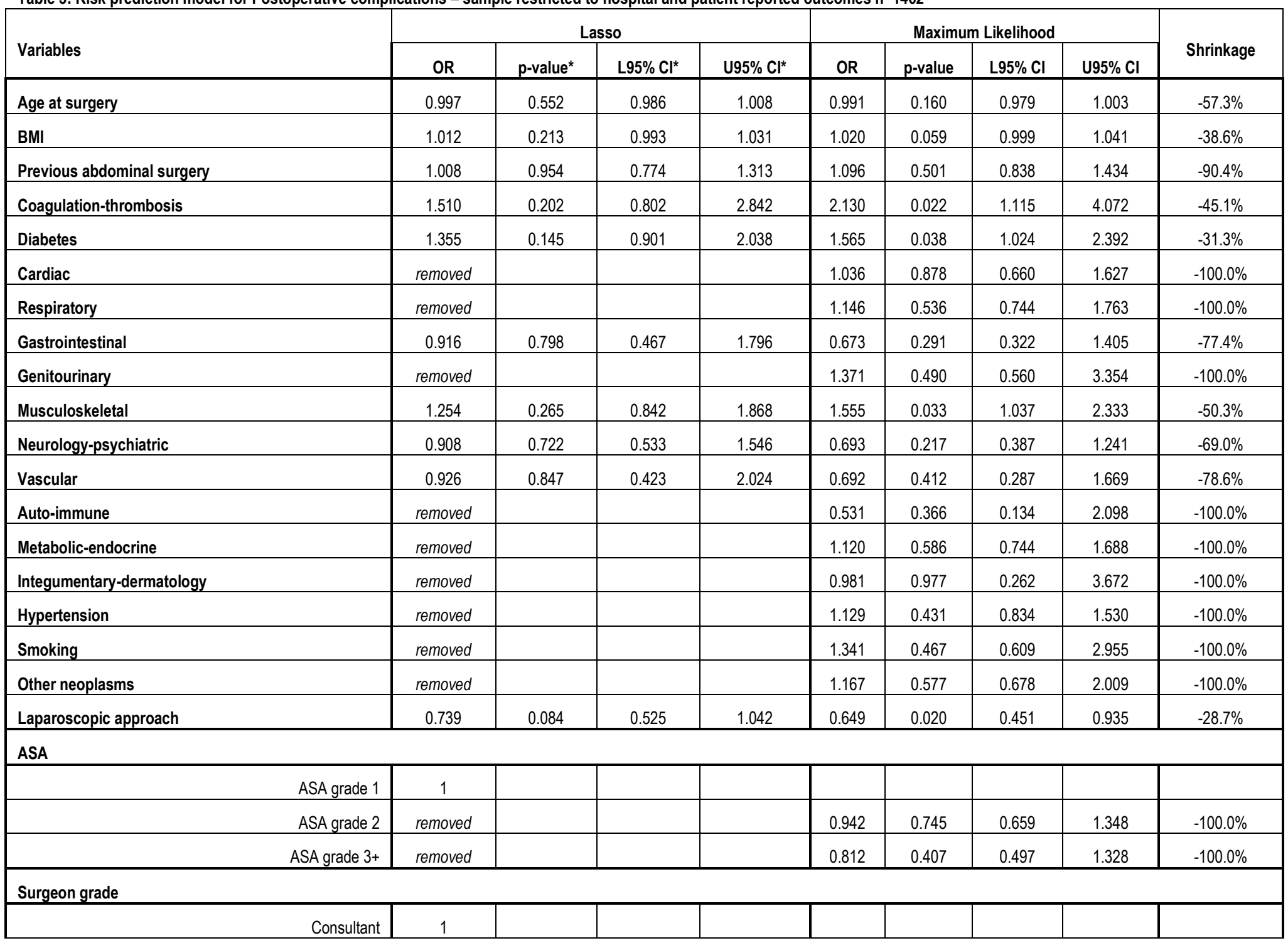




\begin{tabular}{|c|c|c|c|c|c|c|c|c|c|}
\hline Sub-specialty trainee & removed & & & & 0.864 & 0.658 & 0.453 & 1.648 & $-100.0 \%$ \\
\hline General Obstetrics \& Gynaecology Trainee & removed & & & & 0.873 & 0.656 & 0.480 & 1.588 & $-100.0 \%$ \\
\hline \multicolumn{10}{|l|}{ Surgical complexity } \\
\hline Complexity score $1 \& 2$ & 1 & & & & & & & & \\
\hline Complexity score $3 \& 4$ & 1.112 & 0.437 & 0.851 & 1.454 & 1.322 & 0.078 & 0.969 & 1.804 & $-61.8 \%$ \\
\hline Complexity score $5 \& 6$ & removed & & & & 1.054 & 0.819 & 0.670 & 1.659 & $-100.0 \%$ \\
\hline Complexity score $7 \& 8$ & 1.056 & 0.881 & 0.516 & 2.163 & 1.480 & 0.313 & 0.691 & 3.169 & $-83.3 \%$ \\
\hline Complexity score $>8$ & 1.004 & 0.970 & 0.828 & 1.217 & 1.748 & 0.322 & 0.579 & 5.279 & $-90.6 \%$ \\
\hline \multicolumn{10}{|l|}{ Final diagnosis } \\
\hline Ovarian & 1 & & & & & & & & \\
\hline Uterine & 0.982 & 0.911 & 0.719 & 1.343 & 0.951 & 0.790 & 0.658 & 1.376 & $-82.5 \%$ \\
\hline Cervical & 1.606 & 0.094 & 0.923 & 2.794 & 2.099 & 0.016 & 1.148 & 3.836 & $-33.4 \%$ \\
\hline Vulval & 1.779 & 0.030 & 1.056 & 2.999 & 2.274 & 0.003 & 1.311 & 3.943 & $-27.7 \%$ \\
\hline Benign & removed & & & & 1.058 & 0.775 & 0.720 & 1.554 & $-100.0 \%$ \\
\hline Duration of surgery (hrs) & 1.086 & 0.003 & 1.028 & 1.146 & 1.081 & 0.018 & 1.014 & 1.152 & $-6.5 \%$ \\
\hline \multicolumn{10}{|l|}{ Estimated blood loss } \\
\hline$<500 \mathrm{ml}$ & 1 & & & & & & & & \\
\hline $500 \mathrm{ml}-1000 \mathrm{ml}$ & 1.267 & 0.208 & 0.876 & 1.833 & 1.405 & 0.077 & 0.963 & 2.048 & $-32.1 \%$ \\
\hline $1000 \mathrm{ml}-2500 \mathrm{ml}$ & 1.052 & 0.860 & 0.600 & 1.843 & 1.249 & 0.442 & 0.709 & 2.202 & $-76.4 \%$ \\
\hline$>2500 \mathrm{ml}$ & 0.997 & 0.962 & 0.867 & 1.146 & 0.506 & 0.417 & 0.098 & 2.623 & $-86.6 \%$ \\
\hline constant & 0.167 & 0.000 & 0.066 & 0.422 & 0.179 & 0.003 & 0.057 & 0.564 & $-25.5 \%$ \\
\hline
\end{tabular}

* for approximate guidance only. Low albumin not included in the model as only one instance of it amongst 1462 surgeries 
Figure S1: Comparison of observed postoperative complication rates against colour-coded funnel plots. Bright colour-coding and circle markers represent patient-reported statistics and faded colour-coding and square markers represent hospital reported statistics only.

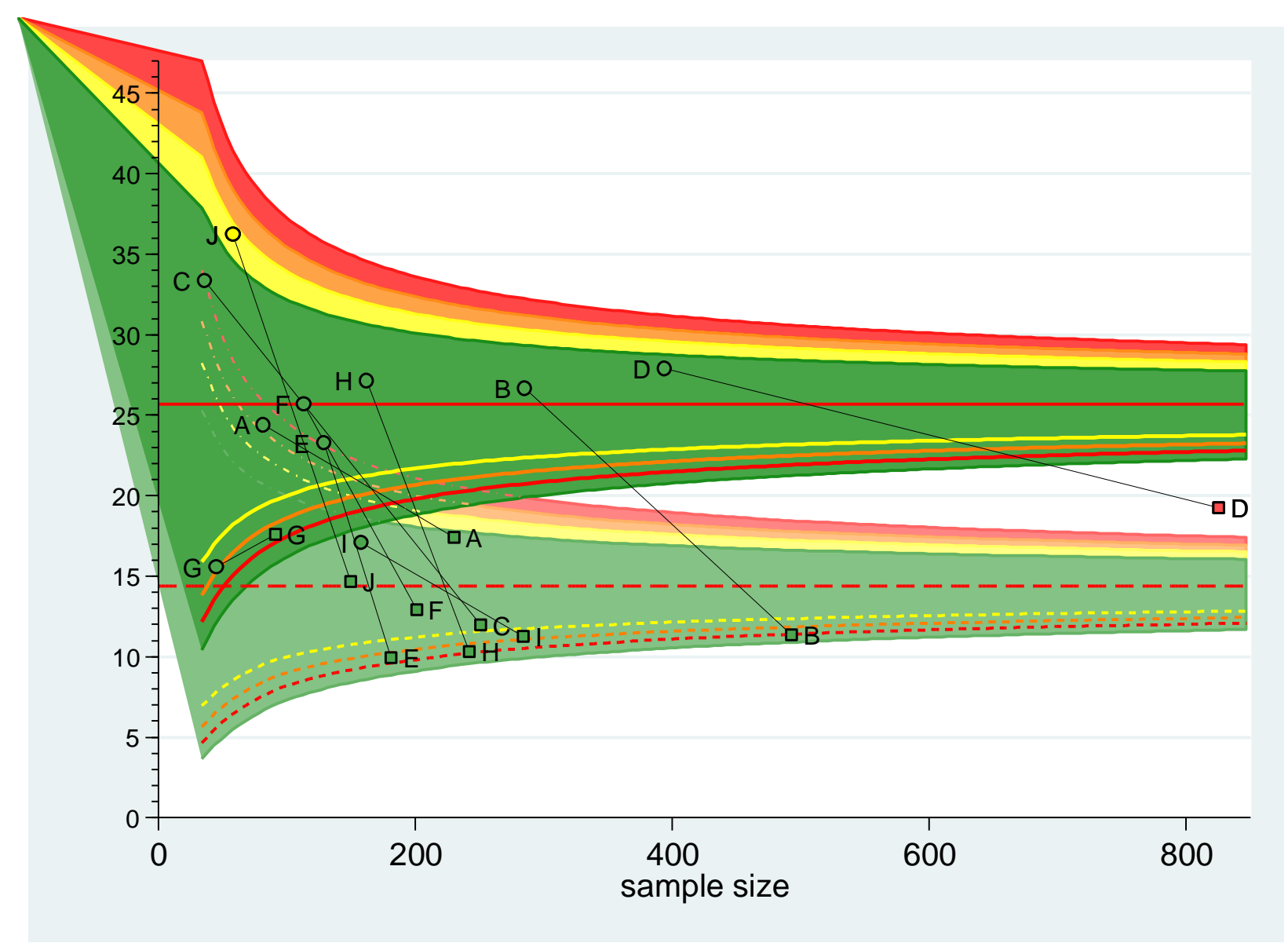




\begin{tabular}{|l|l|}
\hline Table S1: Intra-operative complications \\
\hline Anaesthetic complications & \\
\hline Cardiac & e.g. Cardiac arrythmias, Intra-operative cardic arrest \\
\hline Respiratory & e.g. Aspiration, pneumothorax, pulmonary oedema \\
\hline Allergic reactions & Allergic reactions including anaphylaxis \\
\hline Injury to viscera & \\
\hline Uterine perforation & Perforation of uterus during instrumentation \\
\hline Vascular injury & $\begin{array}{l}\text { Injury to major blood vessel e.g. superior and inferior mesenteric, renal, aorta, } \\
\text { Inferior vena cava, iliacs, femorals, }\end{array}$ \\
\hline Gl tract injury - Stomach & Accidental injury involving complete penetration Into the lumen: Stomach \\
\hline Gl tract injury - Small bowel & Accidental injury involving complete penetration Into the lumen:Small bowel \\
\hline Gl tract injury - Large bowel & Accidental injury involving complete penetration Into the lumen: Large bowel \\
\hline Bladder injury & Accidental bladder injury (full thickness) \\
\hline Ureteric injury & Ligation / Transection / Diathermy burn \\
\hline Intra-operative Haemorrhage & Estimated blood loss >2.5l \\
\hline $\begin{array}{l}\text { Other intra-operative complications } \\
\text { (give details) }\end{array}$ & Other intraoperative complications not included in the list \\
\hline
\end{tabular}




\begin{tabular}{|c|c|}
\hline \multicolumn{2}{|c|}{ Table S2: Post-operative complications } \\
\hline Abscess/Haematoma & Pelvic or abdominal abscess / haematoma \\
\hline \multirow[t]{2}{*}{ Anastomotic leak } & Anastomotic leak: Small bowel \\
\hline & Anastomotic leak: Large bowel \\
\hline lleus & Post op lleus requiring NG tube / Total parental nutrition \\
\hline \multirow{2}{*}{ Bowel obstruction } & Bowel Obstruction - small bowel \\
\hline & Bowel Obstruction - large bowel \\
\hline Bowel perforation & Small / large bowel \\
\hline \multirow[t]{2}{*}{ Bowel - other } & Constipation / Diarrhoea / faecal incontinence/urgency \\
\hline & Urinary retention requiring catheterisation \\
\hline \multirow[t]{2}{*}{ Bladder } & Urinary obstruction \\
\hline & Incontinence- stress / urge \\
\hline Cardiac & $\begin{array}{l}\text { Atrial fibrillation, Myocardial infarction, Cardiac failure \& other cardiac } \\
\text { problems }\end{array}$ \\
\hline DVT & Confirmed DVT on imaging / Doppler \\
\hline $\mathrm{PE}$ & Confirmed PE on imaging \\
\hline \multirow{5}{*}{ Fistula } & Enterocutaneous \\
\hline & Enterovaginal \\
\hline & Vesicovaginal \\
\hline & Ureterovaginal \\
\hline & Other types of fistula \\
\hline Hernia & Hernia as a result of surgery \\
\hline \multirow[t]{2}{*}{ Infection } & $\begin{array}{l}\text { Pyrexia ( }>38.5^{\circ} \mathrm{C} \text { on } 2 \text { separate occasions) after } 48 \text { hours post op requiring } \\
\text { antibiotics or infection confirmed by culture }\end{array}$ \\
\hline & MRSA/ C. difficile \\
\hline \multirow{2}{*}{ Lymphocyst/Lymphoedema } & Lymphoedema \\
\hline & Lymphocyst \\
\hline Neurological & Neuropathic pain/ paraesthesia / nerve palsy \\
\hline
\end{tabular}




\begin{tabular}{|l|l|} 
Psychiatric & $\begin{array}{l}\text { unexpected psychiatric problems postoperatively e.g. Delirium, Psychosis, } \\
\text { Depression and other }\end{array}$ \\
\hline Primary haemorrhage & Haemorrhage within 24 hours of surgery \\
\hline Secondary haemorrhage & Haemorrhage after 24hours of surgery \\
\hline \multirow{2}{*}{ Respiratory } & $\begin{array}{l}\text { Pulmonary oedema, Pneumothorax, Atelectasis, Pleural effusion and other } \\
\text { respiratory problems excluding pneumonia (to be included in infections) }\end{array}$ \\
\hline Ureteric Obstruction & Ureteric obstruction postoperative \\
\hline \multirow{3}{*}{ Wound breakdown } & Wound breakdown: Superficial - skin \& subcutaneous tissue \\
\cline { 2 - 2 } & Wound breakdown: Deep - involving fascia / muscle \\
\cline { 2 - 2 } & Burst abdomen requiring repair under anaesthesia \\
\hline Other & Other postoperative complications not included in the list \\
\hline
\end{tabular}




\begin{tabular}{|c|c|c|c|c|c|c|c|c|c|c|c|c|c|c|c|c|c|c|c|c|c|c|c|c|c|}
\hline & \multicolumn{20}{|c|}{ Hospital } & \multirow{2}{*}{\multicolumn{2}{|c|}{ Overall }} & \multirow{3}{*}{ chi2 } & \multirow{3}{*}{ df } & \multirow{3}{*}{ pvalue } \\
\hline & \multicolumn{2}{|c|}{ A } & \multicolumn{2}{|c|}{ B } & \multicolumn{2}{|c|}{ C } & \multicolumn{2}{|c|}{ D } & \multicolumn{2}{|c|}{ E } & \multicolumn{2}{|c|}{$\mathbf{F}$} & \multicolumn{2}{|c|}{ G } & \multicolumn{2}{|c|}{ H } & \multicolumn{2}{|c|}{1} & \multicolumn{2}{|c|}{ J } & & & & & \\
\hline & $\mathbf{N}$ & $\%$ & $\mathbf{N}$ & $\%$ & $\mathbf{N}$ & $\%$ & $\mathbf{N}$ & $\%$ & $\mathbf{N}$ & $\%$ & $\mathbf{N}$ & $\%$ & $\mathbf{N}$ & $\%$ & $\mathbf{N}$ & $\%$ & $\mathbf{N}$ & $\%$ & $\mathbf{N}$ & $\%$ & $\mathbf{N}$ & $\%$ & & & \\
\hline Intraop complications & 13 & 5.7 & 17 & 3.5 & 10 & 4.0 & 44 & 5.3 & 14 & 7.7 & 4 & 2.0 & 6 & 6.6 & 11 & 4.6 & 8 & 2.8 & 12 & 8.0 & 139 & 4.7 & 16.8 & 9 & 0.0517 \\
\hline Postop complications (hospital reported) & 40 & 17.4 & 56 & 11.4 & 30 & 12.0 & 159 & 19.3 & 18 & 9.9 & 26 & 12.9 & 16 & 17.6 & 25 & 10.3 & 32 & 11.3 & 22 & 14.7 & 424 & 14.4 & 32.0 & 9 & 0.0002 \\
\hline Previous abdominal surgery & 83 & 36.1 & 168 & 34.1 & 64 & 25.5 & 306 & 37.1 & 42 & 23.2 & 84 & 41.8 & 33 & 36.3 & 109 & 45.0 & 117 & 41.2 & 19 & 12.7 & 1,025 & 34.8 & 75.6 & 9 & 0.0000 \\
\hline Low Albumin & 0 & 0.0 & 5 & 1.0 & 0 & 0.0 & 1 & 0.1 & 0 & 0.0 & 0 & 0.0 & 1 & 1.1 & 3 & 1.2 & 0 & 0.0 & 1 & 0.7 & 11 & 0.4 & 17.7 & 9 & 0.0391 \\
\hline Coagulation-thrombosis & 9 & 3.9 & 16 & 3.3 & 12 & 4.8 & 24 & 2.9 & 8 & 4.4 & 9 & 4.5 & 6 & 6.6 & 17 & 7.0 & 9 & 3.2 & 6 & 4.0 & 116 & 3.9 & 11.9 & 9 & 0.2180 \\
\hline Diabetes & 21 & 9.1 & 52 & 10.6 & 30 & 12.0 & 94 & 11.4 & 12 & 6.6 & 15 & 7.5 & 5 & 5.5 & 30 & 12.4 & 18 & 6.3 & 21 & 14.0 & 298 & 10.1 & 17.2 & 9 & 0.0455 \\
\hline Cardiac & 17 & 7.4 & 55 & 11.2 & 32 & 12.8 & 75 & 9.1 & 21 & 11.6 & 23 & 11.4 & 7 & 7.7 & 29 & 12.0 & 27 & 9.5 & 22 & 14.7 & 308 & 10.5 & 10.5 & 9 & 0.3081 \\
\hline Respiratory & 21 & 9.1 & 58 & 11.8 & 21 & 8.4 & 74 & 9.0 & 14 & 7.7 & 20 & 10.0 & 5 & 5.5 & 37 & 15.3 & 21 & 7.4 & 16 & 10.7 & 287 & 9.7 & 16.6 & 9 & 0.0553 \\
\hline Gastrointestinal & 10 & 4.4 & 10 & 2.0 & 6 & 2.4 & 21 & 2.6 & 9 & 5.0 & 17 & 8.5 & 6 & 6.6 & 13 & 5.4 & 3 & 1.1 & 9 & 6.0 & 104 & 3.5 & 35.2 & 9 & 0.0001 \\
\hline Genitourinary & 2 & 0.9 & 5 & 1.0 & 2 & 0.8 & 7 & 0.9 & 6 & 3.3 & 5 & 2.5 & 0 & 0.0 & 14 & 5.8 & 8 & 2.8 & 3 & 2.0 & 52 & 1.8 & 37.2 & 9 & 0.0000 \\
\hline Musculoskeletal & 15 & 6.5 & 23 & 4.7 & 15 & 6.0 & 98 & 11.9 & 13 & 7.2 & 19 & 9.5 & 4 & 4.4 & 34 & 14.1 & 21 & 7.4 & 19 & 12.7 & 261 & 8.9 & 38.7 & 9 & 0.0000 \\
\hline Neurology-psychiatric & 18 & 7.8 & 37 & 7.5 & 12 & 4.8 & 65 & 7.9 & 8 & 4.4 & 15 & 7.5 & 7 & 7.7 & 17 & 7.0 & 22 & 7.8 & 7 & 4.7 & 208 & 7.1 & 6.7 & 9 & 0.6652 \\
\hline Vascular & 4 & 1.7 & 18 & 3.7 & 5 & 2.0 & 18 & 2.2 & 3 & 1.7 & 9 & 4.5 & 4 & 4.4 & 8 & 3.3 & 11 & 3.9 & 6 & 4.0 & 86 & 2.9 & 9.5 & 9 & 0.3916 \\
\hline Infections & 2 & 0.9 & 8 & 1.6 & 2 & 0.8 & 0 & 0.0 & 0 & 0.0 & 0 & 0.0 & 0 & 0.0 & 0 & 0.0 & 1 & 0.4 & 0 & 0.0 & 13 & 0.4 & 24.9 & 9 & 0.0031 \\
\hline Auto-immune & 4 & 1.7 & 8 & 1.6 & 2 & 0.8 & 6 & 0.7 & 3 & 1.7 & 5 & 2.5 & 0 & 0.0 & 2 & 0.8 & 5 & 1.8 & 3 & 2.0 & 38 & 1.3 & 8.5 & 9 & 0.4874 \\
\hline Metabolic-endocrine & 25 & 10.9 & 47 & 9.5 & 25 & 10.0 & 67 & 8.1 & 15 & 8.3 & 27 & 13.4 & 15 & 16.5 & 35 & 14.5 & 35 & 12.3 & 11 & 7.3 & 302 & 10.2 & 18.7 & 9 & 0.0282 \\
\hline Integumentary-dermatology & 4 & 1.7 & 2 & 0.4 & 3 & 1.2 & 6 & 0.7 & 1 & 0.6 & 3 & 1.5 & 0 & 0.0 & 8 & 3.3 & 3 & 1.1 & 0 & 0.0 & 30 & 1.0 & 19.7 & 9 & 0.0199 \\
\hline Hypertension & 61 & 26.5 & 186 & 37.7 & 72 & 28.7 & 257 & 31.2 & 66 & 36.5 & 78 & 38.8 & 19 & 20.9 & 110 & 45.5 & 71 & 25.0 & 53 & 35.3 & 973 & 33.0 & 48.4 & 9 & 0.0000 \\
\hline Smoking & 1 & 0.4 & 25 & 5.1 & 15 & 6.0 & 3 & 0.4 & 5 & 2.8 & 10 & 5.0 & 16 & 17.6 & 14 & 5.8 & 1 & 0.4 & 5 & 3.3 & 95 & 3.2 & 113.7 & 9 & 0.0000 \\
\hline Other neoplasms & 24 & 10.4 & 15 & 3.0 & 6 & 2.4 & 40 & 4.9 & 11 & 6.1 & 6 & 3.0 & 5 & 5.5 & 16 & 6.6 & 19 & 6.7 & 6 & 4.0 & 148 & 5.0 & 27.4 & 9 & 0.0012 \\
\hline Surgeon grade & & & & & & & & & & & & & & & & & & & & & & & 302.4 & 18 & 0.0000 \\
\hline General Obstetrics \& Gynaecology Trainee & 3 & 1.3 & 22 & 4.6 & 3 & 1.3 & 15 & 1.8 & 25 & 15.0 & 3 & 1.5 & 0 & 0.0 & 2 & 0.9 & 30 & 11.1 & 5 & 3.4 & 108 & 3.8 & & & \\
\hline Sub-specialty trainee & 87 & 37.8 & 95 & 19.9 & 65 & 27.2 & 172 & 20.9 & 8 & 4.8 & 3 & 1.5 & 23 & 25.3 & 80 & 35.1 & 35 & 12.9 & 5 & 3.4 & 573 & 20.0 & & & \\
\hline Consultant & 140 & 60.9 & 360 & 75.5 & 171 & 71.6 & 638 & 77.3 & 134 & 80.2 & 192 & 97.0 & 68 & 74.7 & 146 & 64.0 & 206 & 76.0 & 136 & 93.2 & 2,191 & 76.3 & & & \\
\hline Laparoscopic approach & 109 & 47.4 & 40 & 8.1 & 44 & 17.5 & 208 & 25.2 & 8 & 4.4 & 11 & 5.5 & 26 & 28.6 & 62 & 25.6 & 152 & 53.5 & 21 & 14.0 & 681 & 23.1 & 373.2 & 9 & 0.0000 \\
\hline ASA Grade & & & & & & & & & & & & & & & & & & & & & & & 145.5 & 27 & 0.0000 \\
\hline
\end{tabular}




\begin{tabular}{|c|c|c|c|c|c|c|c|c|c|c|c|c|c|c|c|c|c|c|c|c|c|c|c|c|c|}
\hline ASA grade 1 & 44 & 19.2 & 142 & 28.8 & 107 & 43.3 & 194 & 23.5 & 32 & 17.7 & 58 & 28.9 & 23 & 25.3 & 19 & 7.9 & 97 & 34.2 & 38 & 25.3 & 754 & 25.6 & & & \\
\hline ASA grade 2 & 149 & 65.1 & 262 & 53.1 & 115 & 46.6 & 438 & 53.1 & 93 & 51.4 & 101 & 50.3 & 56 & 61.5 & 156 & 65.0 & 138 & 48.6 & 79 & 52.7 & 1,587 & 54.0 & & & \\
\hline ASA grade $3+$ & 36 & 15.7 & 89 & 18.1 & 25 & 10.1 & 193 & 23.4 & 56 & 30.9 & 42 & 20.9 & 12 & 13.2 & 65 & 27.1 & 48 & 16.9 & 33 & 22.0 & 599 & 20.4 & & & \\
\hline Surgical complexity & & & & & & & & & & & & & & & & & & & & & & & 228.9 & 36 & 0.0000 \\
\hline Complexity score $1 \& 2$ & 97 & 42.2 & 149 & 30.2 & 160 & 63.8 & 395 & 47.9 & 118 & 65.2 & 72 & 35.8 & 48 & 52.8 & 124 & 51.2 & 181 & 63.7 & 54 & 36.0 & 1,398 & 47.4 & & & \\
\hline Complexity score $3 \& 4$ & 79 & 34.4 & 199 & 40.4 & 62 & 24.7 & 276 & 33.5 & 38 & 21.0 & 88 & 43.8 & 39 & 42.9 & 79 & 32.6 & 70 & 24.7 & 52 & 34.7 & 982 & 33.3 & & & \\
\hline Complexity score $5 \& 6$ & 41 & 17.8 & 111 & 22.5 & 17 & 6.8 & 105 & 12.7 & 23 & 12.7 & 37 & 18.4 & 3 & 3.3 & 35 & 14.5 & 19 & 6.7 & 39 & 26.0 & 430 & 14.6 & & & \\
\hline Complexity score $7 \& 8$ & 9 & 3.9 & 24 & 4.9 & 5 & 2.0 & 34 & 4.1 & 2 & 1.1 & 4 & 2.0 & 1 & 1.1 & 3 & 1.2 & 6 & 2.1 & 5 & 3.3 & 93 & 3.2 & & & \\
\hline Complexity score $>8$ & 4 & 1.7 & 10 & 2.0 & 7 & 2.8 & 15 & 1.8 & 0 & 0.0 & 0 & 0.0 & 0 & 0.0 & 1 & 0.4 & 8 & 2.8 & 0 & 0.0 & 45 & 1.5 & & & \\
\hline Final diagnosis & & & & & & & & & & & & & & & & & & & & & & & 274.2 & 36 & 0.0000 \\
\hline Ovarian & 94 & 40.9 & 123 & 25.0 & 99 & 39.4 & 305 & 37.0 & 57 & 31.5 & 82 & 40.8 & 27 & 29.7 & 78 & 32.2 & 64 & 22.5 & 60 & 40.0 & 989 & 33.6 & & & \\
\hline Uterine & 70 & 30.4 & 119 & 24.1 & 73 & 29.1 & 243 & 29.5 & 56 & 30.9 & 51 & 25.4 & 37 & 40.7 & 60 & 24.8 & 62 & 21.8 & 49 & 32.7 & 820 & 27.8 & & & \\
\hline Cervical & 18 & 7.8 & 19 & 3.9 & 20 & 8.0 & 82 & 9.9 & 16 & 8.8 & 9 & 4.5 & 5 & 5.5 & 16 & 6.6 & 11 & 3.9 & 11 & 7.3 & 207 & 7.0 & & & \\
\hline Vulval & 3 & 1.3 & 18 & 3.7 & 14 & 5.6 & 69 & 8.4 & 12 & 6.6 & 12 & 6.0 & 4 & 4.4 & 20 & 8.3 & 12 & 4.2 & 12 & 8.0 & 176 & 6.0 & & & \\
\hline Benign & 45 & 19.6 & 214 & 43.4 & 45 & 17.9 & 126 & 15.3 & 40 & 22.1 & 47 & 23.4 & 18 & 19.8 & 68 & 28.1 & 135 & 47.5 & 18 & 12.0 & 756 & 25.6 & & & \\
\hline Estimated blood loss & & & & & & & & & & & & & & & & & & & & & & & 246.2 & 27 & 0.0000 \\
\hline$<500 \mathrm{ml}$ & 155 & 67.4 & 400 & 82.0 & 196 & 78.7 & 709 & 87.4 & 93 & 51.4 & 161 & 80.9 & 69 & 75.8 & 198 & 81.8 & 261 & 93.2 & 93 & 62.0 & 2,335 & 79.9 & & & \\
\hline $500 \mathrm{ml}-1000 \mathrm{ml}$ & 50 & 21.7 & 49 & 10.0 & 33 & 13.3 & 62 & 7.6 & 69 & 38.1 & 23 & 11.6 & 12 & 13.2 & 31 & 12.8 & 11 & 3.9 & 38 & 25.3 & 378 & 12.9 & & & \\
\hline $1000 \mathrm{ml}-2500 \mathrm{ml}$ & 19 & 8.3 & 33 & 6.8 & 16 & 6.4 & 37 & 4.6 & 17 & 9.4 & 15 & 7.5 & 7 & 7.7 & 10 & 4.1 & 7 & 2.5 & 13 & 8.7 & 174 & 6.0 & & & \\
\hline$>2500 \mathrm{ml}$ & 6 & 2.6 & 6 & 1.2 & 4 & 1.6 & 3 & 0.4 & 2 & 1.1 & 0 & 0.0 & 3 & 3.3 & 3 & 1.2 & 1 & 0.4 & 6 & 4.0 & 34 & 1.2 & & & \\
\hline & mean & $s d$ & mean & $s d$ & mean & $s d$ & mean & $s d$ & mean & $s d$ & mean & $s d$ & mean & $s d$ & mean & $s d$ & mean & $s d$ & mean & $s d$ & mean & $s d$ & $\mathbf{F}$ & $d f^{*}$ & pvalue \\
\hline Age at surgery & 58.6 & 13.8 & 59.5 & 15.4 & 58.4 & 15.7 & 60.6 & 15.0 & 63.2 & 12.1 & 63.1 & 13.6 & 57.2 & 12.0 & 63.3 & 16.0 & 59.6 & 14.4 & 64.1 & 14.0 & 60.6 & 14.8 & 5.2 & 9 & 0.0000 \\
\hline BMI & 27.6 & 6.7 & 28.8 & 6.9 & 28.2 & 8.3 & 29.1 & 7.2 & 30.0 & 6.8 & 28.5 & 6.1 & 29.6 & 8.4 & 29.2 & 6.7 & 28.9 & 6.4 & 28.4 & 6.7 & 28.8 & 7.0 & 2.1 & 9 & 0.0304 \\
\hline Duration of surgery (hrs) & 141 & 59.1 & 114 & 53.7 & 151 & 85.1 & 159 & 83.4 & 116 & 52.0 & 122 & 57.0 & 136 & 56.8 & 121 & 58.8 & 101 & 47.9 & 128 & 55.9 & 133.3 & 69.9 & 31.4 & 9 & 0.0000 \\
\hline
\end{tabular}




\begin{tabular}{|c|c|c|c|c|c|c|c|c|c|c|c|c|c|c|c|c|c|c|c|c|c|c|c|c|c|}
\hline & \multicolumn{20}{|c|}{ Hospital } & \multirow{2}{*}{\multicolumn{2}{|c|}{ Overall }} & \multirow{3}{*}{ chi2 } & \multirow{3}{*}{ df } & \multirow{3}{*}{ pvalue } \\
\hline & \multicolumn{2}{|c|}{ A } & \multicolumn{2}{|c|}{ B } & \multicolumn{2}{|c|}{ c } & \multicolumn{2}{|c|}{ D } & \multicolumn{2}{|c|}{$\mathbf{E}$} & \multicolumn{2}{|c|}{$\mathbf{F}$} & \multicolumn{2}{|c|}{ G } & \multicolumn{2}{|c|}{$\mathrm{H}$} & \multicolumn{2}{|c|}{1} & \multicolumn{2}{|c|}{ J } & & & & & \\
\hline & $\mathbf{N}$ & $\%$ & $\mathbf{N}$ & $\%$ & $\mathbf{N}$ & $\%$ & $\mathbf{N}$ & $\%$ & $\mathbf{N}$ & $\%$ & $\mathbf{N}$ & $\%$ & $\mathbf{N}$ & $\%$ & $\mathbf{N}$ & $\%$ & $\mathbf{N}$ & $\%$ & $\mathbf{N}$ & $\%$ & $\mathbf{N}$ & $\%$ & & & \\
\hline Intraop complications & 3 & 3.7 & 10 & 3.5 & 2 & 5.6 & 22 & 5.6 & 10 & 7.8 & 3 & 2.7 & 3 & 6.7 & 8 & 4.9 & 5 & 3.2 & 2 & 3.5 & 68 & 4.7 & 7.1 & 9 & 0.6276 \\
\hline $\begin{array}{l}\text { Postop complications (hospital and patient } \\
\text { reported) }\end{array}$ & 20 & 24.4 & 76 & 26.7 & 12 & 33.3 & 110 & 27.9 & 30 & 23.3 & 29 & 25.7 & 7 & 15.6 & 44 & 27.2 & 27 & 17.1 & 21 & 36.2 & 376 & 25.7 & 14.8 & 9 & 0.0960 \\
\hline Previous abdominal surgery & 23 & 28.1 & 99 & 34.7 & 13 & 36.1 & 141 & 35.8 & 30 & 23.3 & 42 & 37.2 & 14 & 31.1 & 70 & 43.2 & 66 & 41.8 & 9 & 15.5 & 507 & 34.7 & 27.9 & 9 & 0.0010 \\
\hline Low Albumin & 0 & 0.0 & 0 & 0.0 & 0 & 0.0 & 0 & 0.0 & 0 & 0.0 & 0 & 0.0 & 0 & 0.0 & 1 & 0.6 & 0 & 0.0 & 0 & 0.0 & 1 & 0.1 & 8.0 & 9 & 0.5311 \\
\hline Coagulation-thrombosis & 3 & 3.7 & 9 & 3.2 & 2 & 5.6 & 12 & 3.1 & 7 & 5.4 & 3 & 2.7 & 3 & 6.7 & 11 & 6.8 & 5 & 3.2 & 1 & 1.7 & 56 & 3.8 & 8.3 & 9 & 0.4997 \\
\hline Diabetes & 7 & 8.5 & 28 & 9.8 & 5 & 13.9 & 43 & 10.9 & 6 & 4.7 & 7 & 6.2 & 3 & 6.7 & 22 & 13.6 & 13 & 8.2 & 11 & 19.0 & 145 & 9.9 & 15.8 & 9 & 0.0713 \\
\hline Cardiac & 5 & 6.1 & 36 & 12.6 & 10 & 27.8 & 29 & 7.4 & 15 & 11.6 & 12 & 10.6 & 3 & 6.7 & 17 & 10.5 & 18 & 11.4 & 8 & 13.8 & 153 & 10.5 & 20.4 & 9 & 0.0157 \\
\hline Respiratory & 7 & 8.5 & 40 & 14.0 & 3 & 8.3 & 26 & 6.6 & 9 & 7.0 & 11 & 9.7 & 2 & 4.4 & 22 & 13.6 & 10 & 6.3 & 4 & 6.9 & 134 & 9.2 & 19.0 & 9 & 0.0254 \\
\hline Gastrointestinal & 1 & 1.2 & 3 & 1.1 & 1 & 2.8 & 12 & 3.1 & 8 & 6.2 & 7 & 6.2 & 5 & 11.1 & 7 & 4.3 & 1 & 0.6 & 4 & 6.9 & 49 & 3.4 & 26.7 & 9 & 0.0016 \\
\hline Genitourinary & 2 & 2.4 & 2 & 0.7 & 0 & 0.0 & 2 & 0.5 & 4 & 3.1 & 1 & 0.9 & 0 & 0.0 & 11 & 6.8 & 6 & 3.8 & 0 & 0.0 & 28 & 1.9 & 34.3 & 9 & 0.0001 \\
\hline Musculoskeletal & 7 & 8.5 & 17 & 6.0 & 2 & 5.6 & 57 & 14.5 & 8 & 6.2 & 11 & 9.7 & 3 & 6.7 & 28 & 17.3 & 13 & 8.2 & 7 & 12.1 & 153 & 10.5 & 26.4 & 9 & 0.0017 \\
\hline Neurology-psychiatric & 5 & 6.1 & 14 & 4.9 & 1 & 2.8 & 22 & 5.6 & 5 & 3.9 & 7 & 6.2 & 2 & 4.4 & 12 & 7.4 & 16 & 10.1 & 4 & 6.9 & 88 & 6.0 & 8.0 & 9 & 0.5329 \\
\hline Vascular & 2 & 2.4 & 9 & 3.2 & 1 & 2.8 & 6 & 1.5 & 3 & 2.3 & 4 & 3.5 & 2 & 4.4 & 5 & 3.1 & 6 & 3.8 & 2 & 3.5 & 40 & 2.7 & 4.1 & 9 & 0.9046 \\
\hline Infections & 0 & 0.0 & 6 & 2.1 & 0 & 0.0 & 0 & 0.0 & 0 & 0.0 & 0 & 0.0 & 0 & 0.0 & 0 & 0.0 & 1 & 0.6 & 0 & 0.0 & 7 & 0.5 & 20.8 & 9 & 0.0136 \\
\hline Auto-immune & 1 & 1.2 & 3 & 1.1 & 1 & 2.8 & 4 & 1.0 & 3 & 2.3 & 2 & 1.8 & 0 & 0.0 & 1 & 0.6 & 2 & 1.3 & 0 & 0.0 & 17 & 1.2 & 4.4 & 9 & 0.8794 \\
\hline Metabolic-endocrine & 9 & 11.0 & 22 & 7.7 & 4 & 11.1 & 32 & 8.1 & 12 & 9.3 & 14 & 12.4 & 7 & 15.6 & 23 & 14.2 & 22 & 13.9 & 5 & 8.6 & 150 & 10.3 & 11.3 & 9 & 0.2567 \\
\hline Integumentary-dermatology & 2 & 2.4 & 0 & 0.0 & 1 & 2.8 & 1 & 0.3 & 1 & 0.8 & 1 & 0.9 & 0 & 0.0 & 6 & 3.7 & 1 & 0.6 & 0 & 0.0 & 13 & 0.9 & 23.7 & 9 & 0.0048 \\
\hline Hypertension & 25 & 30.5 & 113 & 39.7 & 12 & 33.3 & 127 & 32.2 & 47 & 36.4 & 47 & 41.6 & 12 & 26.7 & 74 & 45.7 & 51 & 32.3 & 17 & 29.3 & 525 & 35.9 & 17.2 & 9 & 0.0458 \\
\hline Smoking & 0 & 0.0 & 14 & 4.9 & 0 & 0.0 & 1 & 0.3 & 2 & 1.6 & 2 & 1.8 & 7 & 15.6 & 9 & 5.6 & 1 & 0.6 & 1 & 1.7 & 37 & 2.5 & 58.1 & 9 & 0.0000 \\
\hline Other neoplasms & 10 & 12.2 & 9 & 3.2 & 1 & 2.8 & 27 & 6.9 & 6 & 4.7 & 3 & 2.7 & 3 & 6.7 & 13 & 8.0 & 10 & 6.3 & 5 & 8.6 & 87 & 6.0 & 15.6 & 9 & 0.0768 \\
\hline Surgeon grade & & & & & & & & & & & & & & & & & & & & & & & 201.5 & 18 & 0.0000 \\
\hline General Obstetrics \& Gynaecology Trainee & 2 & 2.4 & 17 & 6.2 & 1 & 2.9 & 6 & 1.5 & 21 & 17.8 & 3 & 2.7 & 0 & 0.0 & 2 & 1.3 & 19 & 12.5 & 3 & 5.3 & 74 & 5.2 & & & \\
\hline Sub-specialty trainee & 35 & 42.7 & 55 & 20.2 & 16 & 47.1 & 82 & 20.8 & 5 & 4.2 & 1 & 0.9 & 15 & 33.3 & 58 & 38.2 & 21 & 13.8 & 1 & 1.8 & 289 & 20.4 & & & \\
\hline Consultant & 45 & 54.9 & 201 & 73.6 & 17 & 50.0 & 306 & 77.7 & 92 & 78.0 & 107 & 96.4 & 30 & 66.7 & 92 & 60.5 & 112 & 73.7 & 53 & 93.0 & 1,055 & 74.4 & & & \\
\hline Laparoscopic approach & 37 & 45.1 & 21 & 7.4 & 4 & 11.1 & 99 & 25.1 & 8 & 6.2 & 6 & 5.3 & 15 & 33.3 & 43 & 26.5 & 91 & 57.6 & 9 & 15.5 & 333 & 22.8 & 220.3 & 9 & 0.0000 \\
\hline
\end{tabular}




\begin{tabular}{|c|c|c|c|c|c|c|c|c|c|c|c|c|c|c|c|c|c|c|c|c|c|c|c|c|c|}
\hline ASA Grade & & & & & & & & & & & & & & & & & & & & & & & 49.1 & 18 & 0.0001 \\
\hline ASA grade 1 & 10 & 12.4 & 80 & 28.1 & 12 & 33.3 & 90 & 22.8 & 23 & 17.8 & 29 & 25.7 & 9 & 20.0 & 15 & 9.3 & 45 & 28.5 & 17 & 29.3 & 330 & 22.6 & & & \\
\hline ASA grade 2 & 58 & 71.6 & 155 & 54.4 & 21 & 58.3 & 230 & 58.4 & 70 & 54.3 & 64 & 56.6 & 32 & 71.1 & 106 & 65.8 & 82 & 51.9 & 29 & 50.0 & 847 & 58.0 & & & \\
\hline ASA grade $3+$ & 13 & 16.1 & 50 & 17.5 & 3 & 8.3 & 74 & 18.8 & 36 & 27.9 & 20 & 17.7 & 4 & 8.9 & 40 & 24.8 & 31 & 19.6 & 12 & 20.7 & 283 & 19.4 & & & \\
\hline Surgical complexity & & & & & & & & & & & & & & & & & & & & & & & 125.8 & 36 & 0.0000 \\
\hline Complexity score $1 \& 2$ & 36 & 43.9 & 89 & 31.2 & 25 & 69.4 & 179 & 45.4 & 87 & 67.4 & 41 & 36.3 & 22 & 48.9 & 84 & 51.9 & 106 & 67.1 & 21 & 36.2 & 690 & 47.2 & & & \\
\hline Complexity score $3 \& 4$ & 30 & 36.6 & 115 & 40.4 & 9 & 25.0 & 143 & 36.3 & 27 & 20.9 & 46 & 40.7 & 20 & 44.4 & 53 & 32.7 & 38 & 24.1 & 23 & 39.7 & 504 & 34.5 & & & \\
\hline Complexity score $5 \& 6$ & 11 & 13.4 & 60 & 21.1 & 1 & 2.8 & 50 & 12.7 & 13 & 10.1 & 22 & 19.5 & 2 & 4.4 & 23 & 14.2 & 10 & 6.3 & 14 & 24.1 & 206 & 14.1 & & & \\
\hline Complexity score $7 \& 8$ & 3 & 3.7 & 16 & 5.6 & 0 & 0.0 & 15 & 3.8 & 2 & 1.6 & 4 & 3.5 & 1 & 2.2 & 2 & 1.2 & 1 & 0.6 & 0 & 0.0 & 44 & 3.0 & & & \\
\hline Complexity score $>8$ & 2 & 2.4 & 5 & 1.8 & 1 & 2.8 & 7 & 1.8 & 0 & 0.0 & 0 & 0.0 & 0 & 0.0 & 0 & 0.0 & 3 & 1.9 & 0 & 0.0 & 18 & 1.2 & & & \\
\hline Final diagnosis & & & & & & & & & & & & & & & & & & & & & & & 163.5 & 36 & 0.0000 \\
\hline Ovarian & 39 & 47.6 & 70 & 24.6 & 17 & 47.2 & 157 & 39.9 & 39 & 30.2 & 46 & 40.7 & 11 & 24.4 & 54 & 33.3 & 34 & 21.5 & 14 & 24.1 & 481 & 32.9 & & & \\
\hline Uterine & 25 & 30.5 & 68 & 23.9 & 13 & 36.1 & 124 & 31.5 & 40 & 31.0 & 33 & 29.2 & 22 & 48.9 & 41 & 25.3 & 40 & 25.3 & 21 & 36.2 & 427 & 29.2 & & & \\
\hline Cervical & 7 & 8.5 & 7 & 2.5 & 0 & 0.0 & 29 & 7.4 & 12 & 9.3 & 4 & 3.5 & 3 & 6.7 & 10 & 6.2 & 3 & 1.9 & 5 & 8.6 & 80 & 5.5 & & & \\
\hline Vulval & 0 & 0.0 & 14 & 4.9 & 1 & 2.8 & 26 & 6.6 & 7 & 5.4 & 6 & 5.3 & 0 & 0.0 & 10 & 6.2 & 8 & 5.1 & 7 & 12.1 & 79 & 5.4 & & & \\
\hline Benign & 11 & 13.4 & 126 & 44.2 & 5 & 13.9 & 58 & 14.7 & 31 & 24.0 & 24 & 21.2 & 9 & 20.0 & 47 & 29.0 & 73 & 46.2 & 11 & 19.0 & 395 & 27.0 & & & \\
\hline \multicolumn{26}{|l|}{ Estimated blood loss } \\
\hline$<500 \mathrm{ml}$ & 54 & 65.9 & 237 & 84.0 & 24 & 68.6 & 331 & 86.0 & 68 & 52.7 & 91 & 80.5 & 35 & 77.8 & 135 & 83.3 & 148 & 94.9 & 39 & 67.2 & 1,162 & 80.3 & 140.4 & 27 & 0.0000 \\
\hline $500 \mathrm{ml}-1000 \mathrm{ml}$ & 20 & 24.4 & 25 & 8.9 & 8 & 22.9 & 35 & 9.1 & 46 & 35.7 & 14 & 12.4 & 5 & 11.1 & 21 & 13.0 & 4 & 2.6 & 17 & 29.3 & 195 & 13.5 & & & \\
\hline $1000 \mathrm{ml}-2500 \mathrm{ml}$ & 7 & 8.5 & 17 & 6.0 & 3 & 8.6 & 17 & 4.4 & 13 & 10.1 & 8 & 7.1 & 3 & 6.7 & 4 & 2.5 & 4 & 2.6 & 2 & 3.5 & 78 & 5.4 & & & \\
\hline \multirow[t]{2}{*}{$>2500 \mathrm{ml}$} & 1 & 1.2 & 3 & 1.1 & 0 & 0.0 & 2 & 0.5 & 2 & 1.6 & 0 & 0.0 & 2 & 4.4 & 2 & 1.2 & 0 & 0.0 & 0 & 0.0 & 12 & 0.8 & & & \\
\hline & mean & $s d$ & mean & $s d$ & mean & $s d$ & mean & $s d$ & mean & $s d$ & mean & $s d$ & mean & $s d$ & mean & $s d$ & mean & $s d$ & mean & $s d$ & mean & $s d$ & $\mathbf{F}$ & $d f^{*}$ & pvalue \\
\hline Age at surgery & 59.6 & 12.2 & 60.5 & 14.4 & 62.1 & 12.8 & 62.8 & 12.8 & 63.1 & 11.7 & 63.8 & 12.3 & 59.1 & 11.8 & 64.5 & 14.8 & 62.0 & 12.9 & 65.4 & 13.3 & 62.4 & 13.3 & 2.4 & 9 & 0.0107 \\
\hline BMI & 27.8 & 7.8 & 28.8 & 6.7 & 31.1 & 8.8 & 28.5 & 6.8 & 29.7 & 6.6 & 28.3 & 5.5 & 30.2 & 10.0 & 29.2 & 6.9 & 29.1 & 6.3 & 28.6 & 6.3 & 28.9 & 6.9 & 1.2 & 9 & 0.2625 \\
\hline Duration of surgery (hrs) & 148.9 & 60.6 & 110.6 & 51.3 & 146.1 & 91.2 & 161.8 & 80.8 & 110.3 & 51.0 & 121.3 & 55.4 & 138.8 & 53.4 & 122.6 & 56.6 & 95.1 & 42.6 & 114.4 & 34.8 & 128.9 & 66.2 & 23.7 & 9 & 0.0000 \\
\hline
\end{tabular}

